\title{
Energy and Power Considerations in Injection and Optically Pumped Lasers*
}

\author{
AMNON YARIV†, MEMBER, IEEE
}

\begin{abstract}
Summary-The topics of power and energy output from lasers are treated. Both three- and four-level lasers are considered in the pulsed and continuous modes of operation.

The theoretical power and energy estimates are compared, when possible, with experimental data.
\end{abstract}

\section{INTRODLCTION}

$\mathrm{T}$ HIS PAPER is intended primarily as a tutorial review of the subject of power and energy in lasers. It represents a collection of practical information and theories from a variety of sources. These have been presented using a unified point of view in order to bring out the common features which are sometimes lost due to the abundance of individual styles.

We have added, when necessary, a number of derivations and have preceded the main body of the paper with a short review of laser fundamentals in order to minimize the distracting need of referring to other publications.

\section{Quantum Mechanical Backgrotnd}

In Fig. 1 we show a typical energy level diagram such as may be possessed by a laser atom. (The term atom is used generically to describe the active particle, be it an atom, molecule, ion, or electron.) Only some of the low lying levels which participate in the laser action are shown.

The laser emission is due to a transition in which the atom changes from a quantum state 2 with total energy $E_{2}$ to a lower state 1 with energy $E_{1}$. The difference in energy $E_{2}-E_{1}$ is given off as a photon of frequency $h \nu_{12}=E_{2}-E_{1}$.

The following statements are made without proof and will serve as a working basis for the remainder of the paper.

1) An atom in an upper level 2 of a pair of energy levels 2 and 1 will undergo a spontaneous transition to the lower level 1 in a characteristic time $t_{\text {spont }}$ which is independent of the presence of any radiation. We shall assume that $t_{\mathrm{spont}}$ is known. (It has actually been measured in most laser materials.)

2) The reverse process of spontaneous absorption does not exist.

* Received July 19, 1963. The material presented in this paper is based on a lecture given at New York University, N. Y., June 18,1963 .

$\dagger$ Watkins-Johnson Company, Palo Alto, Calif. Formerly with Bell Telephone Laboratories, Inc., Murray Hill, N. J.

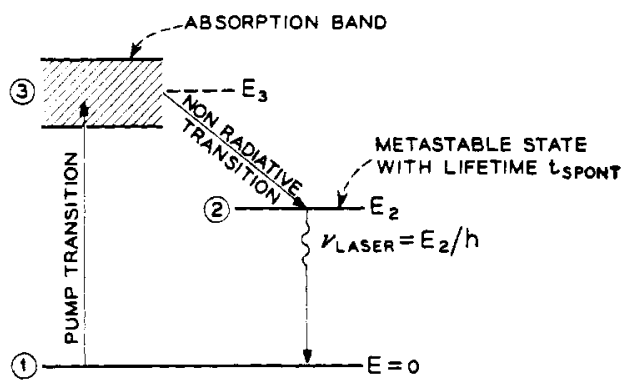

(a)

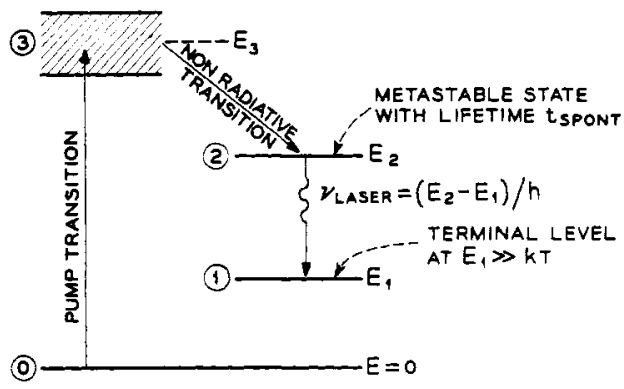

(b)

Fig. 1-(a) Typical energy level diagram of a three-level laser. (b) Typical energy level diagram of a four-level laser.

3) An atom in level 2 (or 1) can undergo, in addition, an induced transition to level 1 (or 2) under the influence of a radiation field of frequency $\nu \sim \nu_{12}$. The transition rate per atom for the induced process is proportional to the energy density and will be denoted as $W_{i}$. It is the same for $2 \rightarrow 1$ or $1 \rightarrow 2$ transitions.

4) The radiation emitted spontaneously, or in response to an inducing field with a uniform spectral density in the vicinity of $\nu_{12}$, will be distributed in frequency, according to a lineshape function $g(\nu)$ with a characteristic width, say at half maximum, of $\Delta \nu$. $\Delta \nu$ can be determined by simple absorption or fluorescence (emission) measurements.

5) The atomic laser system will occupy all or part of the volume of an optical resonator of volume $V$ with characteristic dimensions which are large compared to $\lambda=c / \nu_{12}$. A resonator of this sort will contain [3]

$$
p(\nu)=\frac{8 \pi \nu^{2}}{c^{3}}
$$

modes per unit volume. The number of these 
modes which lie within the atomic linewidth and which can, consequently, exchange energy with it is $\sim p(\nu) \Delta \nu V$ or more exactly

$$
p=\frac{\pi V}{2} p(\nu) \Delta \nu
$$

for a Lorentzian $g(\nu){ }^{1}$

6) The rate of induced emission in to the totality of $p$ modes is

$$
W_{i}=\frac{n_{\mathrm{ph}}}{p t_{\mathrm{spont}}},
$$

where $n_{p h}$ is the total number of photons stored in the $p$ resonator modes.

The decay time constant is $t_{\mathrm{ph}}$ for radiation stored in the resonator, and is equal to $Q / \omega$. It will be referred to as the photon lifetime. The physical significance of $W_{i}$ is that the number of $2 \rightarrow 1$ transitions per second is $W_{i} N_{2}$, while that of $1 \rightarrow 2$ is $W_{i} N_{1}$. The number of photons emitted per second $\phi^{\prime}$ is thus clearly given by

$$
\phi^{\prime}=W_{i}\left(N_{2}-N_{1}\right) \text {. }
$$

\section{The Resonator}

To harness the phenomenon of stimulated emission for laser oscillation, we must feed the stimulated emission back in to the laser medium so that, if we single out a group of photons emitted at a given time by a group of atoms contained in an element of volume $d V \ll \lambda^{3}$, these photons can be made to pass a number of times through the volume $d V$ and have their numbers augmented with each pass according to (3). If this increase is sufficient to overcome other sources of photon loss the density of photons will increase with time thus leading to oscillation. The natural way to provide this feedback and the one suggested originally by Dicke [4] is to incorporate the atomic medium into an optical resonator which usually takes the form of two-plane parallel (or curved) mirrors which cause an essentially plane wave to bounce back and forth between them.

If the characteristic dimensions of the resonator are all large compared to $\lambda$ the mode density is given by (4) regardless of the specific shape of the resonator. Each mode can be characterized by the usual quality factor $Q$.

\section{The Condition for Oscillation Buildup}

We assume that at $t=0$ the resonator contains $n_{\mathrm{ph}}$ photons in the $p$ modes, which according to (2) are on

${ }^{1}$ For a Lorentzian line

$$
g(\nu)=\frac{\Delta \nu}{2 \pi\left[\left(\nu-\nu_{\theta}\right)^{2}+\left(\frac{\Delta \nu}{2}\right)^{2}\right]}
$$

which satisfies the normalization condition

$$
\int_{-\infty}^{+\infty} g(\nu) d \nu=1
$$

"speaking terms" with the atoms. The rate of decay of these photons due to cavity losses is

$$
\left(\frac{d n_{\mathrm{ph}}}{d t}\right)_{\mathrm{loss}}=-\frac{\omega}{Q} n_{\mathrm{ph}}=-\frac{n_{\mathrm{ph}}}{t_{\mathrm{ph}}},
$$

while the stimulated emission causes an increase which according to (3) and (4) is given by

$$
\left(\frac{d n_{\mathrm{ph}}}{d t}\right)_{\text {Eained }}=\frac{n_{\mathrm{ph}}}{p t_{\mathrm{spont}}}\left(N_{2}-N_{1}\right) .
$$

Oscillation build up requires that the gain exceed the resonator losses. This leads to the Schawlow-Townes [1] oscillation condition

$$
n_{c}=\left(N_{2}-N_{1}\right)_{\text {threshold }}=\frac{p t_{\text {spont }}}{t_{\mathrm{ph}}} .
$$

To get an idea of the magnitudes involved here we will calculate, below, $n_{c}$ for a typical pink ruby and a 0.1 per cent $\mathrm{CaF}_{2}: \mathrm{U}^{3+}$ crystal lasers. We shall assume that in each case the distance between reflectors is $10 \mathrm{~cm}$ and the loss per pass is $\sim 2$ per cent so that $t_{\mathrm{ph}} \sim 2 \times 10^{-8} \mathrm{sec}$.

\begin{tabular}{c|c}
\hline $\begin{array}{c}\theta .05 \text { per cent Pink Ruby } \\
\left(N / V=2.5 \times 10^{19} \times \mathrm{cm}^{-3}\right)\end{array}$ & 0.1 per cent $\mathrm{CaF}_{2}: \mathrm{U}^{3+}$ \\
\hline$\Delta \nu\left(300^{\circ} \mathrm{K}\right)=11 \mathrm{~cm}^{-1}$ & $\Delta \nu\left(77^{\circ} \mathrm{K}\right)=15 \mathrm{~cm}^{-1}$ \\
$t_{\text {spunt }}=3 \times 10^{-3} \mathrm{sec}$ & $t_{\text {spont }}=1.3=10^{-4} \mathrm{sec}$ \\
$\nu=14,418 \mathrm{~cm}^{-1}$ & $\nu=3827 \mathrm{~cm}^{-1}$ \\
\hline
\end{tabular}

Substituting these numbers in (7) and using (1) and (2) we get $n_{c / V} \sim 10^{17}$ in ruby and $n_{c / V} \sim 2 \times 10^{14}$ in $\mathrm{CaF}_{2}: \mathrm{U}^{3+}$. The big difference in $n_{c}$ is due to the shorter lifetime and small output frequency of $\mathrm{CaF}_{2}: \mathrm{U}^{3+}$ compared to ruby. Cooling the ruby to $77^{\circ} \mathrm{K}$ reduces $\Delta \nu$ to $\sim 0.2 \mathrm{~cm}^{-1}$, and will thus reduce $n_{c}$ by $\Delta \nu\left(300^{\circ} \mathrm{K}\right) /$ $\Delta \nu\left(77^{\circ} \mathrm{K}\right) \sim 55$.

It is quite instructive to rederive the start-oscillation condition (7) using the traveling-wave point of view. The effort is not totally wasted because many laser considerations, all those involving amplification for instance, can only be treated using this point of view.

We require an expression for $\alpha$ where we assume the presence of inversion $\left(N_{2}-N_{1}\right)$ causes an amplification of the wave intensity as $e^{+\alpha_{z}}$. If we let $I$ be the energy flux in watts $/ \mathrm{m}^{2}$ we have by conservation of energy

$$
\begin{gathered}
\frac{d I}{d z}=\text { Power added by simulated emission per } \mathrm{m}^{3} \\
=\frac{W_{i}\left(N_{2}-N_{1}\right) h \nu}{V}=\frac{n_{\mathrm{ph}}\left(N_{2}-N_{1}\right) h \nu}{p t_{\mathrm{spont}} V}
\end{gathered}
$$

also

$$
I=\frac{\text { Energy Stored in Resonator } \times c}{V}=\frac{n_{\mathrm{ph}} h v c}{V},
$$

where, we recall, $V$ is the resonator volume and $c$ is the 
velocity of light propagation in the laser medium. Combining $(8)$ and $(9)$ we have $[3]^{2}$

$$
\alpha=\frac{d I}{d z} / I=\frac{\left(N_{2}-N_{1}\right)}{c p t_{\text {spout }}} .
$$

If the wave suffers a fractional attenuation in intensity ${ }^{8}$ equal to $\exp (-\gamma)$ in a single pass between the end reflectors, we can write for the start-oscillation condition ${ }^{4}$

$$
e^{\alpha l} e^{-\gamma} \geq 1 \quad \text { or } \quad \alpha \geq \frac{\gamma}{l}
$$

where $l$ is the distance between the reflectors. Since the fractional loss per pass is defined as $\gamma$ and the number of passes per second is $c / l$, it is clear that the decay time constant $t_{\mathrm{ph}}$ and $\gamma$ are related by $t_{\mathrm{ph}}=l / \gamma c$. Substituting this relationship and using (10) leads to $\left(N_{2}-N_{1}\right)>p t_{\text {spont }} / t_{\mathrm{ph}}$ for the ocsillation condition, in agreement with (7).

\section{Three- and Fotr-Level Lasers}

Fig. 1(a) is that of the energy level diagram of a laser in which level 1 is the lowest lying level (which is usually referred to as the ground state). The attainment of inversion $\left(N_{2}>N_{1}\right)$ involves pumping at least half of the ground state population $N$ into level 2. In general, the critical inversion $n_{c}$ as given by (7) is small compared to $N$ so that oscillation results with $N_{2} \approx N / 2$.

Fig. 1(b) illustrates the energy level diagram of a laser in which the terminal level for the laser transition 1 is far enough above the ground state so that its normal population at thermal equilibrium prior to pumping can be neglected, i.e., $N_{1} \ll n_{c}$. This condition results usually when $E_{1}>8 k T$, where $k$ is Boltzmann's constant and $T$ the absolute temperature of the laser medium. Under these conditions oscillation results when $N_{2} \approx n_{c}$.

\section{Considerations of Plmping Thresholds}

The transfer of atoms from the ground state to the metastable (relatively long-lived) level 2 is referred to as pumping and is accomplished by absorption of pumping light from an intense source which causes transitions of atoms from the ground state into the broadband 3. This transition is followed, as shown in Fig. 1 , by a $3 \rightarrow 2$ transition which is usually not asso-

$$
\begin{aligned}
& { }^{2} \text { Using (2) in (10) we have } \\
& \qquad \alpha\left(\nu_{0}\right)=\frac{\left(N_{2}-N_{1}\right) c^{2}}{4 \pi^{2} V \Delta \nu \nu^{2} t_{\mathrm{spont}}}
\end{aligned}
$$

for a Lorentzian lineshape and

for a Gaussian.

$$
\alpha\left(\nu_{0}\right)=\frac{\left(N_{2}-N_{1}\right) c^{2}(\pi \ln 2)^{1 / 2}}{4 \pi^{2} V \Delta \nu \nu^{2} l_{\text {spont }}}
$$

${ }^{3}$ See Yariv and Gordon [3], Section IV for a listing of the main loss mechanisms.

${ }^{4} \gamma$ accounts for losses in the end reflectors as well as in the volume of the resonators. If the two reflectors are not identical we must use $\gamma=\left(\gamma_{1}+\gamma_{2}\right) / 2$ where $\gamma_{1}$ and $\gamma_{2}$ account for the two oppositely directed passes through the resonator. If the losses are due mostly to the mirror reflectance $R$ we have $\exp (-\gamma)=R$ or $\gamma=1-R$ for $\gamma \ll 1$. ciated with the emission of photons, and where the excess energy $\left(E_{3}-E_{2}\right)$ is transmitted to the lattice (phonon emission). In Section VIII we will consider in more detail the role of the absorption band 3 in the pumping process.

The considerations of threshold conditions are different for pulse and $\mathrm{CW}$ lasers and the two cases will be taken up separately.

\section{A. The Threshold Energy Input in a Pulse Laser}

The pumping is provided by a very short pulse of light from an intense flash lamp. We assume that the pulse duration is short compared to $t_{\mathrm{epont}}$ so that the amount of power wasted due to spontaneous transitions (fluorescence) is negligible. The total energy absorbed by the crystal must at least equal that necessary to pump $n_{c}$ atoms to level 2 ;

$$
E_{\min }=\left(N_{2}\right)_{\text {threshold }} \frac{h \nu_{3}}{\eta}
$$

where $h \nu_{3}=E_{3}$ is the mean energy of the absorption band and $\eta$ is the pumping quantum efficiency, i.e., the fraction of atoms that decay to level 2 from level 3 . $\eta$ is a characteristic of the laser material and is often near unity [5].

In a three-level laser we have $\left(N_{2}\right)_{\text {threshold }} \approx N / 2$ so that

$$
E_{\min } \approx \frac{N}{2 \eta}\left(h \nu_{3}\right)
$$

Using the ruby data of Section IV we can calculate (assuming $\eta \approx 1$ ) $E_{\min } / V \sim 3$ joules.

In a four-level laser material we have $\left(N_{2}\right)_{\text {threshold }} \approx n_{c}$ $=p t_{\text {spont }} / t_{\mathrm{ph}}$ so that

$$
E_{\mathrm{min}}=\frac{n_{c}}{\eta}\left(h \nu_{3}\right)=\frac{p t_{\mathrm{spont}}}{\eta t_{\mathrm{ph}}}\left(h \nu_{3}\right) .
$$

Using the data of Section IV for $\mathrm{CaF}_{2}: \mathrm{U}^{3+}$ and assuming $\eta \sim 1$ and $\nu_{3}=11,000 \mathrm{~cm}^{-1}$ (which is the location of the absorption band) we get $E_{\min } / V=10^{-4}$ joules-cm-3. The measured lamp energy inputs to typical ruby and $\mathrm{CaF}_{2}: \mathrm{U}^{3+}$ lasers under typical (inefficient) excitation conditions $[3]$ are: ruby: $\sim 1000$ joules, $\mathrm{CaF}_{2}: \mathrm{U}^{3++}: 1$ joule.

From (13) we see that $E_{\min } / V$ in a three-level laser depends mostly on the concentration of lasing atoms $-N / V$. A reduction of the concentration, therefore, reduces the energy density requirement (as long as $N \gg n_{c}$ ).

The four-level laser, on the other hand, has an $E_{\min }$ which depends, most critically, on the spontaneous lifetime and, through $p$, on $\Delta \nu$. Typical values for $t_{\text {spont }}$ are: $3 \mathrm{msec}$ (ruby), [5], $0.13 \mathrm{msec},[6],\left(\mathrm{CaF}_{2}: \mathrm{U}^{3+}\right) . \Delta \nu$ in ruby is $\sim 0.15 \mathrm{~cm}^{-1}\left(1 \mathrm{~cm}^{-1}=3 \times 10^{10} \mathrm{cps}\right)$ at $77^{\circ} \mathrm{K}$ and $11 \mathrm{~cm}^{-1}$ at $300^{\circ} \mathrm{K} . \Delta \nu$ in $\mathrm{CaF}_{2}: \mathrm{U}^{3+}$ is $\sim 15 \mathrm{~cm}^{-1}$ at $77^{\circ} \mathrm{K}$. Values of these quantities in other laser materials are given in Table $I$ of [3] and in other references listed there. 


\section{B. Threshold Power Input for CW Operation}

The continuous operation of a three-level laser requires the maintenance of, at least, $N / 2$ atoms in level 2. Near threshold we can neglect the shortening of the lifetime due to induced emission so that the number of $2 \rightarrow 1$ transitions per second is $N / 2 t_{\text {spont }}$ which must be compensated by $N / 2 t_{\text {spont }} \eta 1 \rightarrow 3$ transitions per second. The power absorbed by the laser medium is thus

$$
\underset{\substack{\text { min } \\ 3-\text { level }}}{P_{\text {spont } \eta}}=\frac{N h \nu_{3}}{2 l_{\text {spe }}}, N>n_{\boldsymbol{c}} .
$$

The minimum power is inversely proportional to the spontaneous lifetime $t_{\text {spont }}$. As a matter of fact, the lowest reported threshold for $\mathrm{CW}$ operation is that of $\mathrm{CaF}_{2}: \mathrm{Dy}^{2+}$ where $t_{\text {spont }}$ is $\sim 20 \mathrm{msec}$ [3].

Using the same line of reasoning as that leading to (15) we can write

$$
\underset{\substack{\min \\ 4-\text { level }}}{P_{\text {lab }}}=\frac{p\left(h \nu_{3}\right)}{\eta t_{\mathrm{ph}}},
$$

which is seen to be independent of $t_{\mathrm{spont}}$.

\section{Optical Pumping}

In Section VII we have obtained expressions for threshold energies and powers for pulse and $\mathrm{CW}$ laser operation. The next problem is that of achieving enough light from optical pump sources to do the job.

A straightforward optical absorption and fluorescence experiment will yield the following information: 1) The range of frequencies whose absorption results in an efficient fluorescence; 2) The "absorption strength" of the laser medium over the useful absorption regions. As an example we use $\mathrm{CaF}_{2}: \mathrm{U}^{3+}$. A simple experiment [6] was used to establish the fact that useful absorption is provided mostly by three narrow bands centered about $\lambda=0.9 \mu$. An actual absorption spectrograph is shown in Fig. 2. The ordinate gives the absorption coefficient $\beta$ where the incident radiation is attenuated as $e^{-\beta z}$. The peak absorption strength is seen to be (from left to right) $\beta=2.8 \mathrm{~cm}^{-1}, 1.7 \mathrm{~cm}^{-1}$, and $2.2 \mathrm{~cm}^{-1}$. The halfmaximum width $\Delta \nu_{\mathrm{abs}}$ is measured on the abcissa as 8 $\mathrm{cm}^{-1}, 10 \mathrm{~cm}^{-1}$, and $15 \mathrm{~cm}^{-1}$, respectively. The crystal can be subjected to uniform excitation from a lamp which provides, at the crystal surface, an energy flux of $I$ watts $/ \mathrm{cm}^{2}$, assumed constant over the frequency range of interest. Under these conditions the amount of power absorbed by $1 \mathrm{~cm}^{3}$ of material is given by $\int I(\nu) \beta(\nu) d \nu$, which in the case of $\mathrm{CaF}_{2}: \mathrm{U}^{3+}$ discussed above, becomes

$$
\frac{P_{\text {abs }}}{V} \cong(2.8 \times 8+1.7 \times 10+2.2 \times 15) I \times 3 \times 10^{10}
$$

where the factor $3 \times 10^{10}$ converts the $\Delta \nu$ from $\mathrm{cm}^{-1}$ to cps.

To find how much power can be absorbed from a given lamp, we need to know $I(\nu)$. The spectral output characteristics of the lamn are usually provided by the lamp

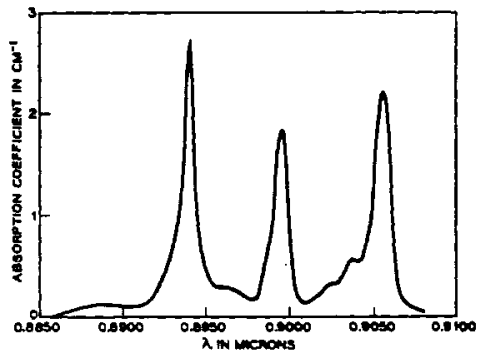

Fig. 2-The absorption characteristics of a 0.1 per cent $\mathrm{CaF}_{2}: \mathrm{U}^{3+}$ in the useful laser excitation region.

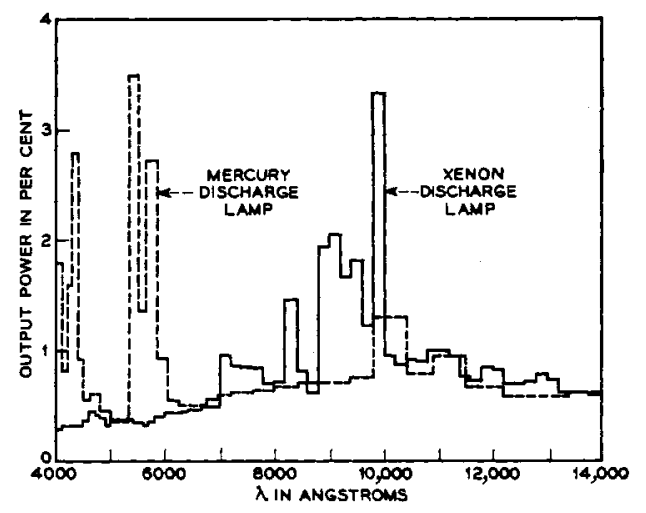

Fig. 3-Spectral output characteristics of two commercial high pressure lamps. One is a mercury lamp the other a xenon lamp. The output is plotted as a fraction of the electrical input to the lamp over certain wavelength intervals (mostly $200 \mathrm{~A}$ ) between $0.4 \mu$ and $1.4 \mu$.

manufacturer. Two such plots are shown in Fig. 3. The engineer must then figure out what fraction of the lamp output is actually incident on the crystal surface in order to get $I(\nu)$. This involves a consideration of the optical system used to illuminate the crystal, and nothing more can be said about it in the present context. The useful absorption must, of course, exceed the minimum requirement as given by (15) and (16).

The practical considerations for flash excitation are identical to those of the CW operation discussed in the preceding paragraph. Some numerical examples of pumping requirements for lasers are to be found in [3]. The value of $\bar{k}$ ( $\beta$ average in the present notation), see [3], is wrong and should be corrected to $\bar{k} \sim 2$. This will reduce the estimate of the necessary useful light flux at the crystal surface to $\sim 1 \mathrm{watt} / \mathrm{cm}^{2}$.

\section{Energy Output from Pulse Lasers}

We treat first the case of the pulsed three-level laser. We shall assume, as in Section VII-A, that the time duration of the pump pulse is short compared to the spontaneous emission lifetime $t_{\text {spont. }}$. When this is the case, we need not worry about the exact shape of the light pulse. The case where the pulse length is long compared to $t_{\text {spont }}$ will be treated in the section on CW lasers. The intermediate case is too complex for a simple analysis and, in general, requires machine computations.

Let us follow the sequence of events which follow upon the triggering of the pump pulse. Absorption of pump photons will cause a buildup of the population of 
level 2 until $N_{2} \approx\left(N+n_{c}\right) / 2$. At this point, the rate of stimulated emission exceeds the loss rate and the very rapid "blow-up" of the energy density in the resonator takes place. This will cause a rapid decrease in $\mathrm{N}_{2}$ due to the large rate of stimulated emission, and thus lead to a near-complete disappearance of the inversion. The details of the competition between the pumping of level 2 by the pump pulse and its emptying by stimulated emission during the oscillation are necessary to explain the shape of the oscillation envelope and the familiar spiking behavior [7]. They are, however, not too important in considering the present problem of energy output. All the energy provided from that point on by the pump is converted almost immediately in to photons at frequency $\nu$. Since any further buildup of $N_{2}$ is prevented by stimulated emission, it is clear that under the circumstances described above, all but $N / 2$ photons are emitted by stimulated emission and constitute "useful output," while the remaining $N / 2$ photons are due to the $N / 2$ atoms present in level 2 when the oscillation ceases and are emitted subsequently by noncoherent spontaneous emission.

If the amount of useful energy absorbed by the laser atoms from the pump source is $E_{\text {in }}$, the number of atoms pumped by this radiation into level 2 is $E_{\text {in }} / \eta h \nu_{3}$. Since all but $N / 2$ transitions result in useful output, we have what is illustrated by Fig. 4.

$$
\text { No. of (useful) photons emitted }=\frac{E_{\text {in } \eta}}{h \nu_{3}}-\frac{N}{2},
$$

and after multiplying by $h \nu$

$$
\underset{\substack{\text { out } \\ 3-\text { level }}}{\nu_{3}}=\frac{\nu}{\nu_{3}} \eta\left[E_{\text {in }}-\frac{N}{2 \eta} h \nu_{3}\right] .
$$

We have shown in Section VII-A that $N h \nu_{3} / 2 \eta$ is the minimum energy necessary to achieve oscillation in a three-level laser so that we can finally write

$$
E_{\text {out }}=\frac{\nu}{\nu_{3}} \eta\left[E_{\mathrm{in}}-E_{\mathrm{min}}\right]
$$

Similar reasoning shows that the same expression applies to the energy output of four-level lasers except that

$$
\underset{\substack{\text { min } \\ 4 \text { leve } 1}}{E_{c}}=n_{c}\left(h \gamma_{3}\right) / \eta
$$

as given by (14).

Fig. 5 shows a plot of $E_{\text {out }}$ vs $E_{\text {in }}$ as obtained by $\mathrm{Li}$ and Sims [8]. Its functional form agrees, except for slight curvature near $E_{\text {out }}=0$, with (17).

In practice, the exact, or of ten even the approximate, values of $E_{\mathrm{min}}, E_{\mathrm{in}}$, and $\eta$ are not known. We do know, however, $\epsilon$, which is the energy input to the flash lamp. Since a given fraction of this energy is absorbed by the laser crystal, we can write instead of (17)

$$
\underset{3-\mathrm{level}}{E_{\mathrm{out}}}=\left[\frac{\epsilon}{\epsilon_{\mathrm{min}}}-1\right] \frac{N h \nu}{2},
$$

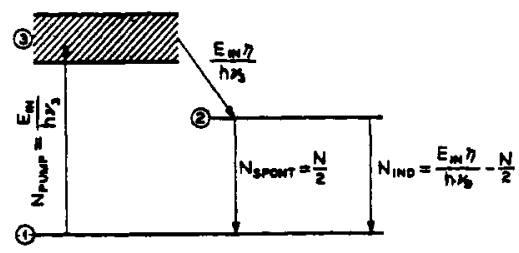

Fig. 4-Detailed balancing for deriving the expression for energy output of a three-level laser. An amount of energy $E_{\text {in }}$ is absorbed from the pump source which is equivalent to $E_{\mathrm{in}_{2}} / h \nu_{3}$ photons. A fraction $\eta$ is transferred to the metastable level 2 . Of these, all but $N / 2$ are emitted by induction and constitute useful output.

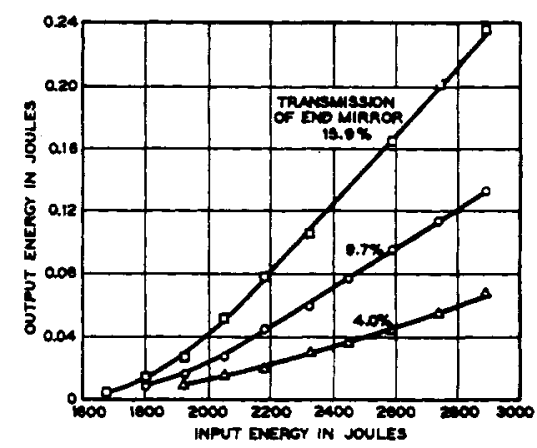

Fig. 5-The energy output of a pulsed ruby laser plotted against the input energy to the flash lamp. The graph is from an article by $\mathrm{Li}$ and Sims [8].

where $\epsilon / \epsilon_{\min }$ is the easily determinable ratio of the actual energy input of the pump lamp to that at threshold.

In the case of a four-level laser we replace $N / 2$ by $n_{c}$ so that

$$
\left.\underset{\substack{\text { out } \\ 4-\mathrm{level}}}{E_{\mathrm{min}}}-1\right] n_{\mathrm{c}} h \nu .
$$

A few comments become pertinent at this stage:

1) Changing the concentration of a three-level laser will not affect $\epsilon_{\min }$, the minimum energy input to the pump source. This is so because the minimum energy $E_{\mathrm{min}}$ is, according to (13), proportional to $N$, but so is the amount of pump light absorbed for a given incident flux, since the absorption coefficient is proportional to concentration. The result is that the reduced need for energy, if $N$ is made smaller, is balanced by a reduced absorption, and the threshold energy input to the pump source $\boldsymbol{\epsilon}_{\mathrm{min}}$ is unchanged.

2) A change of $N$ will cause proportional change in $E_{\text {out }}$ (three-level case) according to (18). Statements 1) and 2) are valid only for crystals which are optically thin at $\nu_{3}$ so that the illumination by the pump light can be considered uniform throughout the volume of the laser crystal and where $n_{c} \ll N$.

3) In a four-level laser $\epsilon_{\min }$ is inversely proportional to concentration (again in optically thin crystals). This is because the minimum energy absorbed by the crystal at threshold $E_{\min }$ does not depend on concentration according to (14). However, the amount of energy absorbed from a given light 
flux is proportional to the optical density of the crystal, and hence, to concentration. A reduction, for example, of $N$ by $\times 2$ must be compensated by a doubling of the flux, and hence $\epsilon_{\mathrm{min}}$, to supply the constant $E_{\min }$. If $\epsilon \gg \epsilon_{\min }$, it follows from the above and (19) that $E_{\text {out }}$ is proportional to $N$. This point may, be demonstrated more easily if we write (19) as $E_{\text {out }}=\left[E / E_{\min }-1\right] n_{c} h \nu$, since $E_{\text {min }}$ is independent of $N$, while $E$ is proportional to it.

4) As for comparing the power output of three- and four-level lasers, let us consider the hypothetical case of three- and four-level lasers having the same $n_{c}$, the same (or equivalent) absorption characteristics and the same quantum efficiency $\eta$. In such a case, the ratio of $\epsilon_{\min }$ of the three-level laser to that of the four-level laser is equal to $N / 2 n_{c}$. If we assume, as we had all along, that $n_{c} \ll N$, using (18) and (19) we get

$$
\frac{\left(E_{\text {out }}\right)_{3-\text { level }}}{\left(E_{\text {out }}\right)_{4-\text { level }}}=1-\frac{\left(\epsilon_{\text {min }}\right)_{3-\text { level }}}{\epsilon},
$$

so that if $\epsilon \gg \epsilon_{\min }$, one can obtain nearly as much power from a three-level as from a four-level laser.

The largest energy outputs reported to date are around 500 joules and were obtained from Glass: $\mathrm{Nd}^{3+}$ at $1.06 \mu$ (a four-level system) and from three-level ruby lasers.

The approaches used to date to obtain more energy are: 1) Using larger crystal rods with larger and brighter lamps for pumping, and 2) more efficient geometries for concentrating the light from the lamp onto the crystal. Major improvement could come from the discovery of laser materials with very broad and intense absorption bands. At the moment, only a small fraction of the energy output of the pump lamps is absorbed.

\section{High Power Pulsed Lasers}

In the case described in the previous section we were concerned mostly with the total energy output of the laser. Nothing was said about the power. To get an idea about the magnitude of the power involved we may assume that the length of the pump pulse is long compared to the resonator ringing time $t_{\mathrm{ph}}$, which is the case in the normally used pump sources. When this condition applies, we expect the output pulse to last for approximately the duration of the pump pulse (assuming the latter is well above threshold). The average power output is then given by $E_{\text {out }} / \tau_{\text {pulse }}$. Assuming a typical high-power laser with $E_{\text {out }}=10$ joules, and $\tau_{\text {pulse }}=0.5 \mathrm{msec}$ will have $P_{\text {ave }} \sim 2 \times 10^{4}$ watts.

Two techniques have been used to increase the peak power level available from lasers.

The first involves the use of nonregenerative (i.e., no feedback) laser amplifiers. These have been demonstrated and discussed by Geusic and Scovil [9]. Gains of $\sim 10 \mathrm{db}$ per stage are easily attained.

The second method is that of $Q$ switching. Its application to lasers was first proposed by Hellwarth [10] and

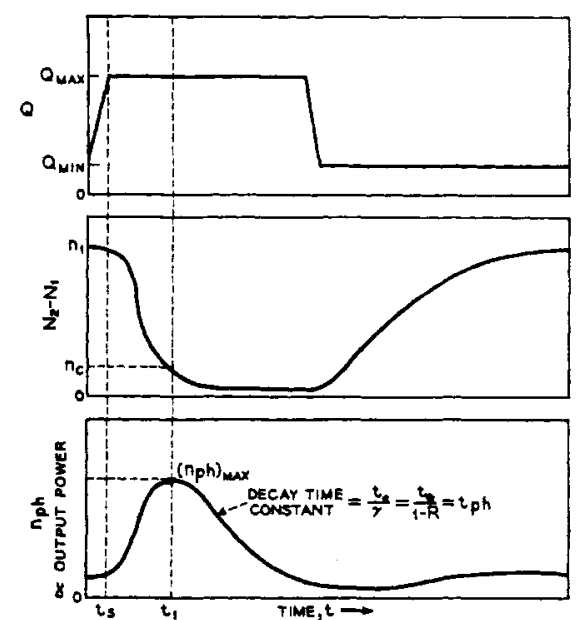

Fig. 6-A qualitative plot of $Q$, the population inversion $\left(N_{2}-N_{1}\right)$ and the power output (which is proportional to the number of photons in the resonator), in a single cycle of a $Q$-switched laser. The pump is assumed to be on continuously.

demonstrated by Hellwarth and McClung [11]. In a $Q$-switched laser the $Q$ of the laser is degraded drastically for the duration of the pump pulse. This, according to (7) (remembering that $t_{\mathrm{ph}}=Q / \omega$ ), makes it possible to obtain an inversion $N_{2}-N_{1}$ far greater than the critical inversion corresponding to the normal $Q$. The restoration of the high $Q$ will then cause a very fast "blow-up" of stored energy in the resonator which is due to the large amplification factor [see (10)].

The theory of this device was worked out by Hellwarth [10] and the reader is referred to his original paper for a fuller discussion. We will, however, rederive the expression for the peak power output.

The cycle of events for a near ideal $Q$-switched laser is shown in Fig. 6. The pump pulse has just ended at $t=0$, the inversion is at its peak value $n_{1}$ and the $Q$ has its minimum value $Q_{\mathrm{min}}$. By $t=t_{s}$ the $Q$ is restored to its original high value. If the switching time $t_{s}$ is short enough there is a negligible change in the inversion during this period. ${ }^{5}$ The blow-up of radiation occurs between $t_{s}$ and $t_{1}$. By the time $t=t_{1}$ the inversion has disappeared (or nearly disappeared) by stimulated emission and the energy stored by the excited atoms in level 2 has been converted into photons. If $t_{1}$ is small compared to $t_{\mathrm{ph}}$, which is the case, most of the emitted photons will still be present in the resonator at $t=t_{1}$. Since the instantaneous power output is given by $h \nu n_{\mathrm{ph}} / t_{\mathrm{ph}}$, the maximum power $P_{\max }$ output is available at this point. To calculate $P_{\max }$ we assume that the pumping cycle succeeded in pumping all of the $N$ atoms into level 2 so that $n_{1}=N$. This, in the case of a three-level laser, will result in $\sim N / 2$ stimulated transitions ${ }^{6}$ so that the number of photons stored in the resonator at $t=t_{1}$ is given by

- As a matter of fact this is exactly the criterion used, [10], to discover how long a $t_{s}$ can be used and yields, for typical cases, $t_{3}<10^{-9}$ secs.

The $\frac{1}{2}$ factor accounts for the fact that after $N / 2$ transitions the inversion is zero, and no further increase in photon density by stimulated emission is possible. 


$$
\left(n_{\mathrm{ph}}\right)_{\max } \sim \frac{N}{2}
$$

The peak power output is

$$
P_{\max } \sim \frac{N(h \nu)}{2 t_{\mathrm{ph}}} .
$$

In a four-level laser the number of downward transitions which cause a complete $\left(n_{1}=N\right)$ inversion to disappear is $\sim N$ so that $P_{\max }$ is given by

$$
P_{\max } \sim \frac{N(h \nu)}{t_{\mathrm{ph}}}
$$

If the pumping does not result in a complete inversion, we replace $N$ in (22) and (23) by $N_{\max }-N / 2$ and $N_{\max }$, respectively, where $n_{\max }$ is the inversion just before the oscillation pulse. $n_{\max } \sim 0.6 N$ in ruby is typical.

Using the numerical example of Section IV we have $N=2.5 \times 10^{19} \mathrm{~V}, t_{\mathrm{ph}}=2 \times 10^{-8} \mathrm{sec}$. Using $V=1 \mathrm{~cm}^{3}$ we get $P_{\max }=165 \mathrm{Mw}$. Peak power outputs in excess of 50 $\mathrm{Mw}$ were reported by a number of laboratories. The $Q$ spoiling was achieved by using Kerr cells in combination with polarizers in a manner like that suggested originally by Hellwarth [10] and by a variety of other means.

\section{Power Output in Continuods Operation}

This subject has been treated in detail elsewhere [3]-[12]. It would be of interest, however, to show how the tools developed here can yield the same results in a less rigorous but far simpler manner.

Consider first the three-level laser. Above threshold we must, according to Section VI, main tain $\approx N / 2$ atoms in level 2 . As a result, there are $N / 2 t_{\text {spont }}$ transitions per second which do not contribute to the oscillation power and are "wasted." The total number of transitions per second, under steady-state conditions, must equal the total number of absorbed pump photons multiplied by the quantum efficiency $\eta$. This gives Rate of $2 \rightarrow 1$ transitions - Rate of $1 \rightarrow 2$ transitions $=P_{\text {in }} \eta / h \nu_{3}$, where $P_{\text {in }}$ is the useful power at frequency $\nu_{3}$ absorbed by the laser atoms. Total number of useful transitions is thus given by $P_{\text {in }} \eta / h \nu_{3}-N / 2 t_{\text {spont. }}$. The net oscillation power is

$$
P_{\text {out }}=\left[\frac{P_{\text {in }} \eta}{h \nu_{3}}-\frac{V}{2 t_{\text {spont }}}\right] h \nu,
$$

or using the definition of $P_{\min }$ in (15),

$$
P_{\text {out }}=\left[P_{\text {in }}-P_{\min }\right] \eta \frac{\nu}{\nu_{3}} .
$$

Another useful form results if we rewrite (24) as

$$
P_{\text {out }}^{3-\text { levels }}=\left[\frac{P_{\text {in }}}{P_{\min }}-1\right] \frac{N h \nu}{2 t_{\text {spont }}} .
$$

This is a very useful form since $P_{\text {in }} / P_{\min }$ is the factor by which threshold is exceeded. The equation is thus of use in cases where the ratio of $P_{\text {in }} / P_{\mathrm{min}}$ has been deter-

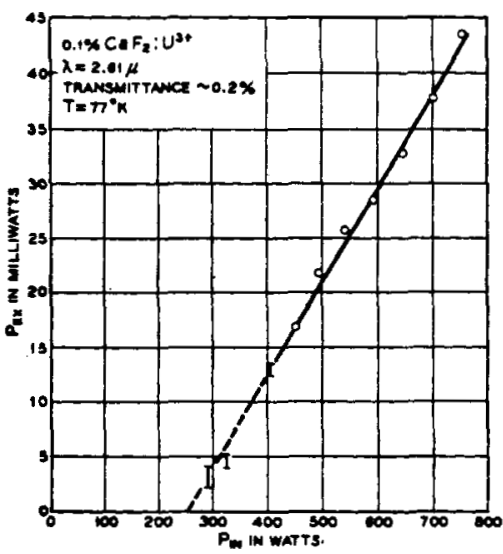

Fig. 7-A plot of the output power vs the electrical energy input to a xenon lamp in a $\mathrm{CW} 0.1$ per cent $\mathrm{CaF}_{2}: \mathrm{U}^{3+}$ laser. The mirror transmittance at $2.61 \mu$ is 0.2 per cent and corresponds to severe undercoupling $(S<1 / 20)$.

mined operationally. For more preliminary investigations, one must estimate $P_{\text {in }}$ along the lines considered in Section VIII and use (24).

The same reasoning applies to a four-level laser, except that the critical population of level 2 is not $N / 2$, but $n_{e}$. We can write directly

$$
P_{\text {out }}=\left[P_{\text {in }}-P_{\text {min }}\right] \eta \frac{\nu}{\nu_{3}}=\left[\frac{P_{\text {in }}}{P_{\text {min }}}-1\right] \frac{n_{c} h \nu}{t_{\text {spont }}} .
$$

To check the validity of (26) we performed a power measurement on a $\mathrm{CW} 0.1$ per cent $\mathrm{CaF}_{2}: \mathrm{U}^{3+}$ laser at $77^{\circ} \mathrm{K}$ with the following parameters:

$$
\begin{aligned}
\lambda_{\text {out }}= & 2.61 \mu \\
L= & 3 \mathrm{~cm} \\
V= & 0.2 \mathrm{~cm}^{3} \\
t_{\mathrm{pb}}= & 1.9 \times 10^{-9} \mathrm{sec} \\
& \left(\begin{array}{c}
\text { loss per pass } \sim 8 \text { per cent, index }- \\
\text { of refraction } \sim 1.4
\end{array}\right), \\
\Delta V= & 15 \mathrm{~cm}^{-1}\left(77^{\circ} \mathrm{K}\right) \\
t_{\text {spon }}= & 0.13 \mathrm{msec} .
\end{aligned}
$$

Using this data we get

$$
n_{c} / V=2.2 \times 10^{15} \mathrm{~cm}^{-3} \text { and } n_{c} h \nu / t_{\text {spont }} \sim 0.26 \mathrm{w} .
$$

The crystal was coated with two dielectric mirrors having a transmission of 3.5 per cent and losses of $\sim 0.4$ per cent. The loss per pass in the resonator, exclusive of the mirrors, was estimated as $\sim 4$ per cent. With the power input 3.6 times above threshold, ${ }^{7}$ i.e., $P_{\text {in }} / P_{\min }$ $=3.6$, we measured $P_{\text {out }}=1$ watt. Since approximately half the output was dissipated in the resonator, the total output was $\sim 2$ watts. The calculated value is $P_{\text {out }}$ $=3.6 \times 0.26=0.67$ watt. This is fair agreement considering the uncertainties in the parameters used in the theory and some uncertainty in the calibration of the infrared power measuring equipment.

A plot of the power output of a $\mathrm{CaF}_{2}: \mathrm{U}^{3+}$ laser vs the input power is shown in Fig. 7. The observed data are in qualitative agreement with (26).

${ }^{7} P_{\text {in }}$ was $\sim 900$ watts and was obtained from a short arc xenon lamp in an elliptical pumping geometry. 


\section{Optimum Cotpling}

The problem of optimum coupling in $\mathrm{CW}$ lasers was considered by Yariv and Gordon [3]. We shall merely state the results for completeness. If we define the coupling coefficient $S$ as the ratio of reflector transmittance to the average loss per pass $^{8}$ we have for a fourlevel laser

$$
S_{\text {optimum }}=-1+\sqrt{P_{\text {in }} / P_{\text {min }}},
$$

where $P_{\min }$ is the threshold pump power at zero transmittance. It follows that near threshold $S_{\text {optinum }} \ll 1$. This distinguishes this case from that of passive circuits where maximum power output results when $S=1$.

In a three-level laser we have (see Appendix)

$S_{\text {optimum }}=-1+\sqrt{1-\left(\frac{N}{n_{c 0}}\right)\left(\frac{1-P_{\text {in }} / P_{\text {min }}}{1+P_{\text {in }} / P_{\text {min }}}\right)}$

where $n_{c 0}$ is the value of $n_{c}$ for zero coupling $(s=0)$. Since $N \gg n_{c 0}$, the three-level laser must, in practice, nearly always be overcoupled $(S>1)$. If we assume $N=100 n_{\mathrm{c} 0}, S_{\text {optimum }}>1$ as soon as $P_{\text {in }}>1.06 P_{\min }$, i.e., as soon as the threshold is exceeded by more than 6 per cent. In the four-level laser, according to (27), overcoupling is necessary only for $P_{\text {in }}>4 P_{\text {in }}$.

To illustrate the method we will derive the optimum coupling condition for a four-level pulsed laser. [Eq. (27) was derived for $\mathrm{CW}$ operation.]

The total power output is given by

$$
E_{\text {out }}=\left[\frac{E_{\text {in }}}{E_{\min }}-1\right] n_{c} h \nu,
$$

which is (19) where the ratio $\epsilon_{\mathrm{in}} / \boldsymbol{\epsilon}_{\min }$ is replaced by the equal quantity $E_{\text {in }} / E_{\min }$. Using (14) for $E_{\min }$ and (7) for $n_{c}$ and ignoring a proportionality constant we get

$$
E_{\mathrm{out}} \propto\left[\frac{E_{\mathrm{in} \eta}}{p t_{\mathrm{spont}} h \nu_{3}}-\frac{1}{t_{\mathrm{ph}}}\right],
$$

where $E_{\text {out }}$ refers to the total output from the laser atoms. But only the fraction $\left(1+S^{-1}\right)^{-1}$ is available externally as useful output, so that

$$
E_{\text {ext }} \propto\left[A-\frac{1}{t_{\mathrm{ph}}}\right] \frac{1}{1+S^{-1}},
$$

with $A=E_{\text {in }} \eta /\left(p t_{\text {spont }} h \nu_{3}\right)$. The last step is to write

$$
\frac{1}{t_{\mathrm{ph}}}=\frac{1}{t_{\mathrm{ph}}{ }^{0}}(1+S) \text {, }
$$

where $t_{\mathrm{ph}}{ }^{0}$ is the unloaded $(S=0)$ photon lifetime and is considered as a constant. Eq. (21) becomes

$$
E_{\text {external }} \propto\left[A-\frac{1}{t_{\mathrm{ph}}{ }^{0}}(1+S)\right] \frac{1}{1+S^{-1}} ;
$$

setting $\partial E_{\text {ex }} / \partial S=0$ yields

$$
S_{\text {optimum }}=-1+\sqrt{E_{\mathrm{in}} / E_{\mathrm{min}}},
$$

which is the same as the expression for the continuous operation (27) with $P_{\text {in }}$ and $P_{\mathrm{m} \text { in }}$ being replaced by $E_{\text {in }}$ and $E_{\mathrm{min}}$. We can similarly use (28) for the pulsed threelevel laser after replacing $P$ by $E$.

\section{Injection Lasers}

The problem of power output from injection lasers [13]-[15] can be treated by the methods developed in the preceding sections. The electrons which are injected from the $n$ side of the junction into the $p$ side can be considered, prior to recombination, to be equivalent to atoms in the excited state 2 (see Fig. 1), while the role of the lower state 1 is played by the acceptor or valence band states. We can then adopt directly the results of Section IV and obtain an expression for the critical current at threshold [16].

$$
I_{c}=\frac{8 \pi e \nu^{2} d A \Delta \nu}{\xi \eta c^{2}}\left(L+L^{\prime}\right)
$$

where $\nu$ is the laser frequency, $\Delta \nu$ is the spectral width of the recombination output, $c$ is the velocity of light in the medium, $A$ is the diode area, $\xi$ is an inversion parameter which at low temperatures $\left(T<20^{\circ} \mathrm{K}\right.$ for GaAs) is near unity, $L$ is the exponential $\left(e^{-L z}\right)$ loss constant of the medium for radiation at $\nu$, and $L^{\prime}$ is the effective increase in $L$ due to losses at the end surfaces. $d$ is the "depth of penetration" of the electromagnetic radiation away from the junction plane [16]$[18]$ and is $\sim 10 \mu$.

An increase of the injection current beyond $I_{c}$ causes a buildup of the radiation density in the optical resonator and a consequent shortening, by stimulated emission, of the recombination lifetime. The additional injections, above threshold, give rise to oscillation power output on the basis (assuming $\eta=1$ ) of one photon per injected electron. We can write immediately the expression for the power output as

$$
P_{\text {out }}=\frac{\left(I-I_{c}\right)}{e} h \nu \text {. }
$$

$h \nu / e$ in injection lasers is nearly equal to the value of the energy gap (expressed in $e V$ ). Most of the useful semiconductors have energy gaps that fall between 0.5 volt and 3 volt so that for biasing currents of a few amperes we may expect power output in the watt region.

\section{Appendix-Optimum Coupling in Three Level Lasers}

The derivation of the optimum coupling condition cannot proceed from the approximate expression given in (24) and requires an exact solution of the problem.

Referring to the energy levels of Fig. 4 we can write the following rate equations:

$$
\begin{aligned}
\frac{d N_{1}}{d l}= & W_{21} N_{2}+W_{i} N_{2}-W_{i} N_{1}-A P N_{1}+A P N_{3} \\
& +N_{3} W_{31} \\
\frac{d N_{2}}{d t}= & W_{21} N_{2}-W_{i} N_{2}+W_{i} N_{1}+N_{3} W_{32}
\end{aligned}
$$




$$
\frac{d N_{3}}{d t}=A P N_{1}-A P N_{3}-Y_{3}\left(W_{32}-W_{31}\right)
$$

Where $W_{i}$ is the induced transition rate per atom for a $2 \leftrightarrow 1$ transition, $W_{i j}$ is the spontaneous transition rate for an $i \rightarrow j$ transition ( $W_{21}=1 / t_{\text {spont }}$ where $t_{\text {spont }}$ is the lifetime of level 2) and $A P$ is taken as the induced transition rate connecting the ground state 1 and the absorption band 3 due to a pump field of intensity $P$.

Using the steady state solutions of $(34)-(36)$ and taking the total population $N_{1}+N_{2}+N_{3}=N$ we get after some manipulations

$$
N_{2}-N_{1}=N \frac{\left(A p \eta-W_{21}\right)}{2 W_{i}+W_{21}+A p \eta}
$$

where $\eta=W_{32} /\left(W_{32}+W_{31}\right)$ is the absorption quantum efficiency and is equal to the fraction of the absorbed pump quanta that transfers its energy to the metastable level 2 .

Under study state oscillation conditions $\left(N_{2}-N_{1}\right)$ cannot exceed the value

$$
n_{c}=\frac{p t_{\mathrm{spont}}}{t_{\mathrm{ph}}}=\frac{p}{W_{21} t_{\mathrm{ph}}}
$$

as given by (7). By equating these two expressions for $N_{2}-N_{1}$ we obtain the following expression for $W_{i}$ :

$$
W_{i}=\frac{N A P \eta W_{21} t_{\mathrm{ph}}}{2 p}-\frac{N W_{21} t_{\mathrm{ph}}}{2}-\frac{p}{2}-\frac{A P \eta}{2 W_{21}} .
$$

Using the relation $P_{\text {out }}=\left(N_{2}-N_{1}\right) h \nu W_{i}$ and (7) in (38) we get

$$
\frac{P_{\text {out }}}{h \nu}=\left(\frac{\nu-n_{c}}{2}\right) A P \eta-\left(\frac{\nu+n_{c}}{2}\right) W_{21} .
$$

The first term on the right side of (39) represents the total power absorbed by $\left(N-n_{c}\right) / 2$ atoms in the ground state, while the second term accounts for the power wasted through spontaneous emission by $\left(N+n_{c}\right) / 2$ atoms in the metastable level. If we neglect $n_{c}$ in comparison to $N$ (39) becomes identical to (24).

To derive the optimum coupling condition we take advantage of the coupling constant $S$ introduced in Section XII. In terms of $S$ we can write $n_{c}=n_{c 0}(1+S)$ where $n_{c 0}$ is the critical inversion for zero coupling. Remembering that only the fraction $\left(1+S^{-1}\right)^{-1}$ is available externally we get from (39)

$$
\begin{aligned}
\frac{2 P_{\mathrm{ext}}}{h \nu}= & A P \eta\left[N-n_{c 0}(1+S)\right] \frac{1}{1+S^{-1}} \\
& -W_{21}\left[N+n_{c 0}(1+S)\right] \frac{1}{1+S^{-1}} .
\end{aligned}
$$

Maximization of $P_{\text {ext }}$ with respect to $S$ leads to

$$
S_{\mathrm{opt}}{ }^{2}+2 S_{\mathrm{opt}}+\frac{N}{n_{c 0}}+1-\frac{2 X}{n_{c 0}} \frac{A P \eta}{A P \eta+W_{21}}=0,
$$

and

$$
S_{\text {opt }}=-1+\sqrt{\frac{N}{n_{c 0}} \frac{A P \eta / W_{21}-1}{A P \eta / W_{21}+1}} .
$$

This is the desired result. A slightly more useful form results if we introduce the quantity $P / P_{\min }(P R$ in [3] which is the factor by which the pump power exceeds its threshold value. This ratio is given from (39) as

$$
P / P_{\min }=\frac{A P \eta}{W_{2}} \frac{N-n_{c}}{N+n_{c}},
$$

which when substituted into (42) yields

$$
S_{\mathrm{opt}}=-1+\sqrt{-\frac{N}{n_{c}} \frac{\left(1-P / P_{\min }\right)}{\left(1+P / P_{\min }\right)}+\frac{4 P / P_{\min }}{\left(1+P / P_{\min }\right)^{2}}}
$$

to first order in $n_{c} / N$. For $N \gg n_{c}$ we can write

$$
S_{\mathrm{opt}}=-1+\sqrt{1+\left(\frac{N}{n_{c}}\right)\left(\frac{P / P_{\min }-1}{P / P_{\mathrm{itin}}+1}\right)}
$$

which is (28).

\section{ACKNOTLLEDGMENT}

The author wishes to thank R. W. Hellwarth for useful information included in this paper, and $\mathrm{D}$. $\mathrm{H}$. Olson for capable experimental assistance in the $\mathrm{CW}$ laser experiments and for the use of Fig. 3.

\section{REFERENCES}

[1] A. L. Schawlow and C. H. Townes, "Infrared and optical masers," Phys. Rev., vol. 112, pp. 1940-1949; December, 1958.

[2] S. O. Heavens, "Optical masers," Appt. Optics Suppl. 1: Optical Masers, pp. 1-23; December, 1962.

[3] A. Yariv and J. P. Gordon, "The laser," Proc. IEEE, vol. 51, pp. 4-29; January, 1963.

[4] R. H. Dicke, "Molecular Amplification and Generation Systems and Methods," U. S. Pat. No. 2,851,652; September 9, 1958.

[5] T. H. Maiman, "Optical and microwave optical experiments in ruby," Phys. Rev. Lett., vol. 4, pp. 564-566; June, 1960.

[6] G. D. Boyd, R. J. Collins, S. P. S. Porto, A. Yariv, and W. A. Hargreves, "Excitation, relaxation, and continuous master action in the 2.613 micron transition of $\mathrm{CaF}_{2}: \mathrm{L}^{3+}$ masers," Phys. Rev. Lett., vol. 8, pp. 269-272; April, 1962.

[7] R. J. Collins, D. F. Nelson, A. L. Schawlow, W. Bond, C. G. B. Garrett, and W. Kaiser, "Coherence, narrowing, directionality, and relaxation oscillations in the light emission from ruby," Phys. Rev. Lett., vol. 5, pp. 303-305; October, 1960.

[8] T. Li and S. D. Sims, "A calorimeter for energy measurements of optical masers," Appl. Optics, vol. 1, pp. 325-329; May, 1962.

[9] J. E. Geusic and H. E. D. Scovil, "A unidirectional travelingwave optical maser," Bell Sys. Tech.J., vol. 41, pp. 1371-1398; July, 1963.

[10] R. W. Hellwarth, "Control of fluorescent pulsations," in "Advances in Quantum Electronics," J. R. Singer, Ed., Columbia University Press, New York, N. Y., pp. 334-342; 1961.

[11] R. W. Hellwarth and F. J. McClung, "Giant optical pulsations from ruby," Bull. Am. Phys. Soc., vol. 5, p. 414; November, 1961.

[12] A. Yariv, "Theory of Power Output and Optimum Coupling in Laser Oscillators," Presented at the 3rd Internat'l Conf. on Quantum Electronics, Paris, France; 1962.

[13] R. N. Hall, G. E. Fenner, J. D. Kingsley, T. J. Soitys, and R. O. Carlson, "Coherent light emission from GaAs junctions," Phys. Rev. Litt., vol. 9, pp. 366-367; November, 1962.

[14] M. I. Nathan, IV. P. Dumke, G. Burns, F. H. Dills, and G. Lasher, "Stimulated emission of radiation from GaAs $p-n$ junctions," Appl. Phys. Lett., vol. 1, pp. 62-6t; November, 1962.

[15] T. M. Quist, R. J. Keyes, W. E. Krag, B. Lax, A. L. Mcllhorter, R. H. Rediker, and H. J. Zeiger, "Semiconductor maser of GaAs," Appl. Phys. Lett., vol. 1, pp. 91-93; December, 1962.

[16] A. Yariv and R. C. C. Leite, "Dielectric-waveguide mode of light propgation in p-n junctions," Appl. Phys. Lett., vol. 2, pp. 55-57; February, 1963. Also: A. Yariv and R. C. C. Leite, "Conditions for spectral narrowing and coherent emission in recombination radiation," Proc. 3rd Internat'l Conference on Quantum Electronics, Paris, France; (to be published).

[17] W. L. Bond, B. G. Cohen, R. C. C. Leite, and A. Yariv, "Observation of the dielectric-waveguide mode of light propagation in p-n junctions," Appl. Phys. Lett., vol. 2, pp. 57-59; February, 1963.

[18] R. C. C. Leite and A. Yariv, "On mode confinement in $p-n$ junctions," Proc. IEEE (Correspondence), vol. 51, pp. 10351036; July, 1963. 